\title{
Channel estimation of OFDM in C-band communication systems under different distribution conditions
}

\author{
Heba Abdul-Jaleel Al-Asady ${ }^{1}$, Hassan Falah Fakhruldeen², Mustafa Qahtan Alsudani ${ }^{3}$ \\ ${ }^{1}$ Computer Technical Engineering Department, College of Technical Engineering, The Islamic University, Najaf, Iraq \\ ${ }^{2,3}$ Computer Techniques Engineering Department, Faculty of Information Technology, Imam Ja'afar Al-sadiq University, \\ Baghdad, Iraq \\ ${ }^{1,2}$ Electrical Engineering Department, College of Engineering, University of Kufa, Kufa, Iraq
}

\begin{tabular}{|c|c|}
\hline Article Info & ABSTRACT \\
\hline Article history: & \multirow{9}{*}{$\begin{array}{l}\text { Orthogonal frequency division multiplexing (OFDM) is a transmission system } \\
\text { that uses multiple orthogonal carriers that are sent out at the same time. OFDM } \\
\text { is a technique for mobile and wireless communication that has high-efficient } \\
\text { frequency utilization, high data-rate transmission, simple and efficient } \\
\text { implementation using the fast Fourier transform (FFT) and the inverse fast } \\
\text { Fourier transform (IFFT), and reduces inter symbol interference (ISI) by } \\
\text { inserting cyclic prefix (CP). One of the most important approaches in an } \\
\text { OFDM system is channel estimation. In this paper, the orthogonal frequency } \\
\text { division multiplexing system with the Rayleigh channel module is analyzed for } \\
\text { different areas. The proposed approach used large numbers of subcarriers to } \\
\text { transmit the signals over 64-QAM modulation with pilot add channel } \\
\text { estimation. The accuracy of the OFDM system is shown in the measuring of } \\
\text { the relationships of peak power to the noise ratio and bit error rate. }\end{array}$} \\
\hline Received Apr 30, 2021 & \\
\hline Revised Jul 7, 2021 & \\
\hline Accepted Aug 11, 2021 & \\
\hline Keywords: & \\
\hline Channel estimation & \\
\hline FFT & \\
\hline OFDM system & \\
\hline QAM modulation & \\
\hline
\end{tabular}

This is an open access article under the CC BY-SA license.

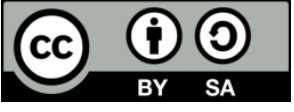

Corresponding Author:

Heba Abdul-Jaleel Al-Asady

Computer Technical Engineering Department

College of Technical Engineering

The Islamic University, Najaf, Iraq

Email: en.he22@gmail.com

\section{INTRODUCTION}

The expanding necessity for high-piece rate advanced versatile interchanges has impelled the presence of orthogonal frequency-division multiplexing (OFDM) for accomplishing great execution at a high pace of information transmission. In different correspondence frameworks for transmission of information, the orthogonal frequency division multiple access is a various access plans are chosen as a physical layer interface for cutting edge remote correspondence frameworks like IEEE [1]. OFDM is a transmission strategy that utilized numerous bearers in the symmetrical structure to transmit the back rubs all the while [2]. In remote correspondence systems especially in OFDM, Synchronization is liberal to make a nature of organization and high-transmission limit without disregarding the critical property that is subcarrier evenness [3]. The best possible structure of the mind-boggling genuine OFDM framework is dissected in compelling and proficient usage, fast Fourier transform (FFT) and the inverse fast Fourier transform (IFFT), embeddings cyclic prefix (CP) to diminish the impact of inter-symbol interference (ISI) and multi-channel fading [2]. Each subcarrier is tweaked with an ordinary advanced regulation plan, (for example, QPSK, 16QAM, and so on.) [4]. In that capacity OFDM has a generally longer image term than single bearer frameworks (because of the lower bit pace of sub-channels) which makes it extremely invulnerable to quick channel blurring and drive clamor, channel adjustment is utilized to remunerate twisting of the sign at the recipient [5], [6]. This 
paper gives considers the impact of utilizing a Rayleigh direct in the OFDM framework in more than three sorts of regions. The conclusive outcomes and the exhibition of the framework are estimated as far as capacity to clamor proportion and number of bits mistakes rate.

\section{TRANSCEIVER OFDM SYSTEM IMPLEMENTATION}

In the OFDMA framework, numerous symmetrical sub-carrier signals are firmly dispersed and covered for transmission of data. These symmetrical subs-carrier don't meddle with one another and give roughness to the inter-symbol interference and blurring of the channel (ISI) [1], [7]. The input information to be transmitted is first conveyed by the sub-carrier signals toss the balancing procedure which utilized here 64-PSK at the low image rate. A complete transceiver OFDM system is described as shown in Figure 1.

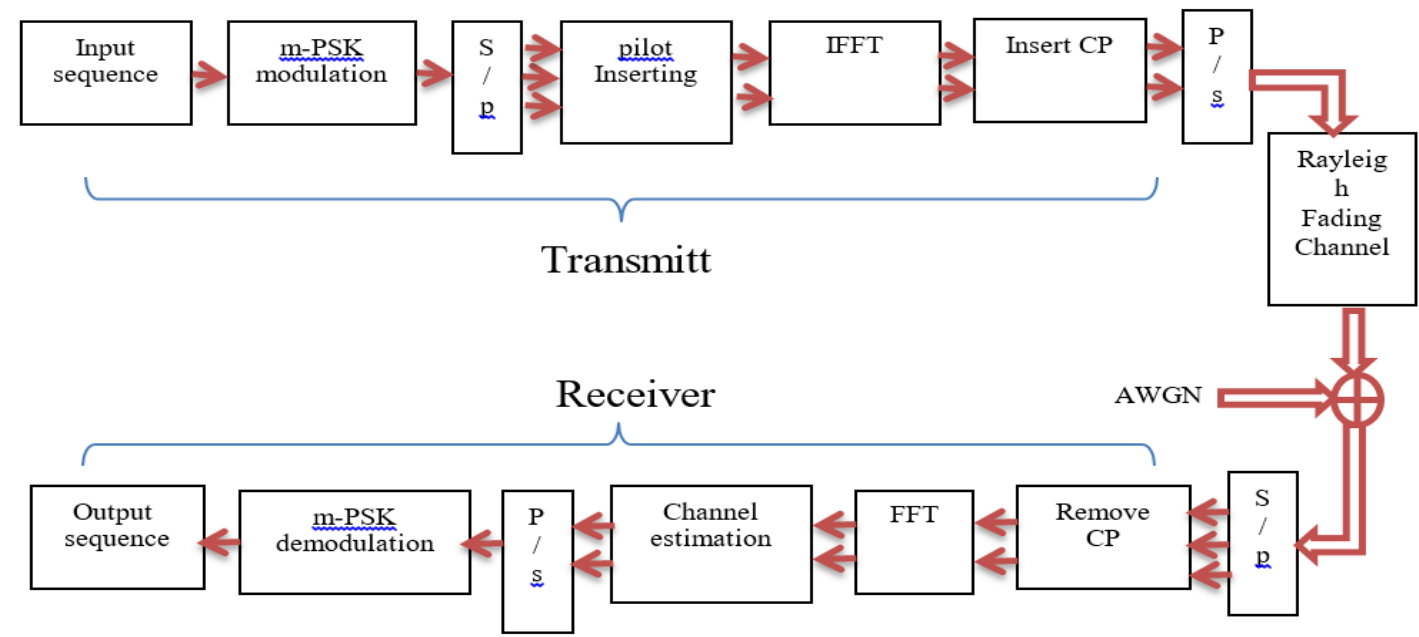

Figure 1. Block diagram for an OFDM system

This tweak strategy has his own arrangement of favorable circumstances which are offered to the correspondence framework [8]-[10], besides; partition the information after regulated it into various equal information streams to embed the pilot data on each stream and afterward utilizes IFFT activity which rearranges it in the beneficiary by applying FFT, inter-symbol interference (ISI) can be disposing of by including an unfilled gatekeeper interim (GI) between two rehashed symbols [11]. The most generally utilized technique to include GI is cyclic prefix $(\mathrm{CP})$ which is a reiteration of the finish of the image which is put toward the beginning of the image. The cyclic prefix is added to stay away from between square obstructions and protect symmetry between sub-channels [12], [13]. By and large, the length of the cyclic prefix is picked with the end goal that the watchman interim is longer than or proportional to the concede spread of the channel; these cyclic are expelled at the beneficiary side. Sequential to-resemble, just as corresponding to-sequential transformation, channel estimation, lastly; advanced to simple and simple to the computerized change process, every one of these activities, happen to the sign when utilized OFDM framework.

\section{CHANNEL}

The channel is demonstrated as a period area complex-baseband move work, which may then be convolved with the transmitted sign to decide the sign at the collector side. additive white Gaussian noise: This is an occupy model in which the primary crippling to correspondence is a straight development of wideband or foundation clamor a predictable spooky thickness and a Gaussian scattering of bounty. The model does not represent blurring, recurrence selectivity, obstruction, non-linearity or scattering. In any case, it produces basic scientific models that are useful for picking up data on the fundamental conduct of a framework before these other marvels are considered [14]-[16]. Rayleigh fading: This is an authentic model to see the impact of blurring on the spread of radio sign in a situation, as used by remote contraptions. Rayleigh obscuring models acknowledge that the enormity of a sign that has experienced such a transmission medium (additionally called a correspondences channel) will change discretionarily or on the other hand 
obscure; as indicated by a Rayleigh circulation, the winding piece of the aggregate of two uncorrelated Gaussian unpredictable components [17]. Rayleigh obscuring is viewed as a reasonable model for tropospheric and ionospheric signal inducing similarly as the effect of enthusiastically created urban circumstances on radio signs [18]. Rayleigh blurring is best appropriate when there is no prevailing engendering along with a view between the transmitter and collector [19], [20].

\section{CHANNEL ESTIMATION}

The channel estimation strategies for OFDM frameworks dependent on the pilot course of action are explored. The channel estimation dependent on brush type pilot course of action is considered through various calculations for both evaluating channels at pilot frequencies and inserting the channel [21]-[23]. The estimation of the channel at pilot frequencies depends on LS and LMS while the channel insertion is finished utilizing straight interposition, second solicitation inclusion, low-pass presentation, spline cubic interjection, also, time-space addition. The time-space introduction is gotten by breathing easy area through inverse discrete Fourier transform (IDFT), zero cushioning and returning to recurrence space through discrete Fourier transform (DFT) [24], [25]. Also, Pilots are sent in all the sub-transporters of the principal image of each square and channel estimation is performed by utilizing LS estimation.

\section{SIMULATION RESULTS}

In the sections we presented the simulation results of OFDM system by bit error rate (BER) and signal to noise ratio SNR, which can be defined as the rate on which the blunder has happened during the information transmission in advanced correspondence. BER is fundamentally influenced by the commotion, twisting, and weakening in the channel. the sign capacity to the degree of commotion power is SNR. On the off chance that the SNR proportion is high then it implies it contains increasingly helpful data and less boisterous information. Figures will show the estimated samples behind the transmitted signals in a different area. For the algorithm considered in Figure 1, the studies are held in three different areas (bad urban, typical urban, and Rural area) as shown in Figures 2, 3 and 4. The channel adopted is a Rayleigh fading with 6 tap time delay one with 64- QAM modulations. Input data was modulating onto 128 subcarriers; The parameters of OFDM symbol duration is chosen as 16 cyclic prefix length, the 1e-3 timing period of the channel, 64 numbers of symbols for QAM modulation and the OFDM frames $=1000$ frames. Figures show the simulation results for BER versus SNR for each area alone and finally the comparison of all results with each other's to see the adequacy of the estimator on the OFDM system with varied regions. The LS estimator is seemed, by all accounts, to be the major figuring and gives standard results. Used with fundamentally all the plans of channel estimation, the LS estimator will be conveyed as an extent between the data course of action and the yield. The compression of the above figures is shown in Figure 5 to see the the differnces of them. Finally, to focuses on the better performance under AWGN and Rayleigh channel fading can show in Figure 6 which is the comparession study of a bit error rate of them.

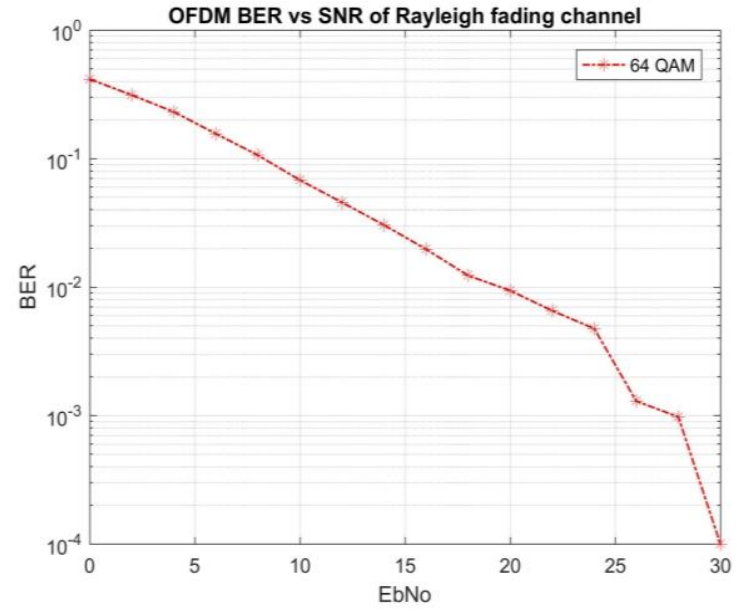

Figure 2. BER of OFDM model for Rural area

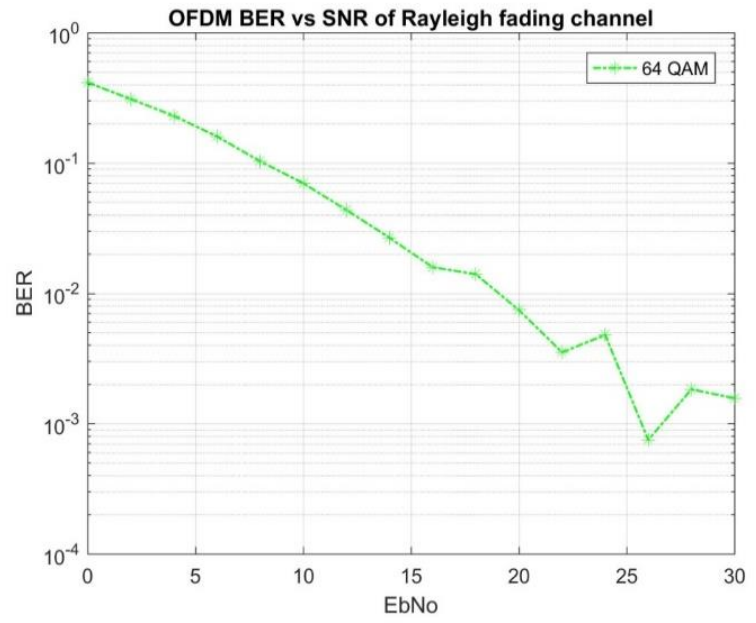

Figure 3. BER of OFDM model for typical Urban area 


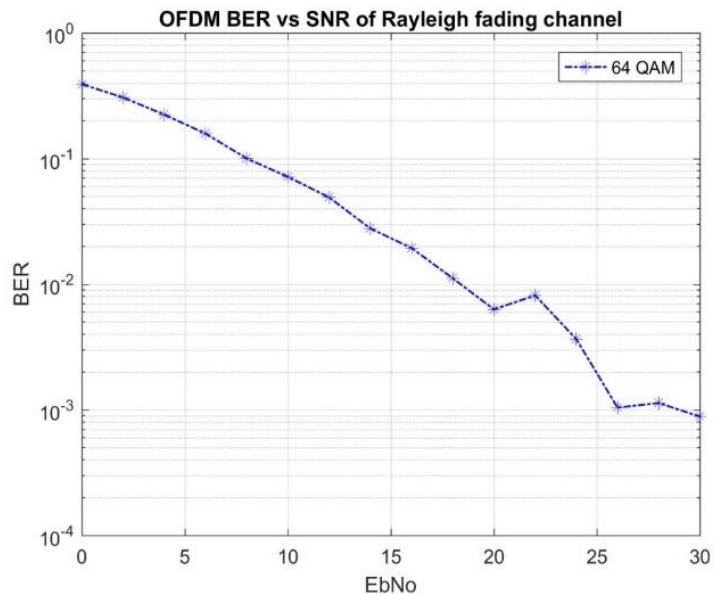

Figure 4. BER of OFDM model for the bad Urban area

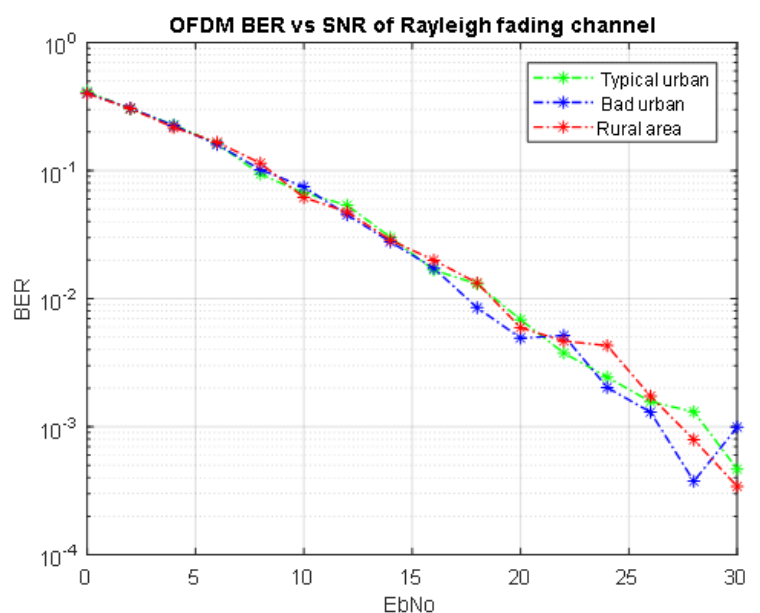

Figure 5. comparession of the above figures with each other of BER of OFDM model

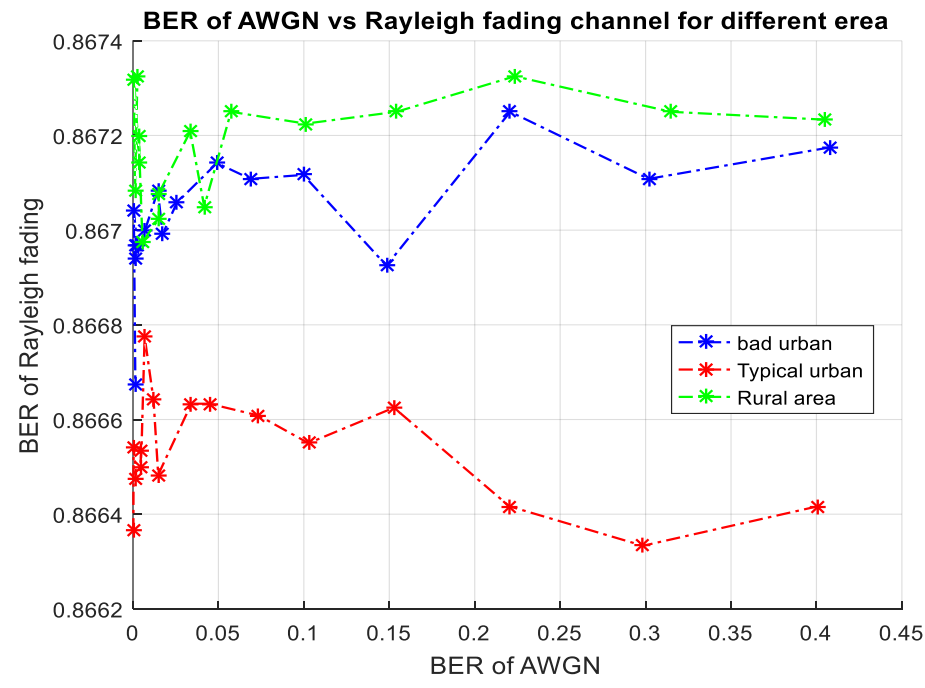

Figure 6. Comparession the bit error rate of AWGN and rayleigh channel fading for the areas

\section{CONCLUSION}

In this paper, we present an OFDM system with the pilot-aided channel estimation techniques over different areas (bad urban, typical urban, and rural areas). The LS algorithm utilizes channel prior information on clamor difference and covariance of the channel, this calculation can acquire a relatively good SNR and BER performance, we can conclude that the QAM gives better performance under AWGN and Rayleigh channel fading. In all three areas, we note that at a small value of the signal/ noise ratio the numbers of errors are high and reached nearly 10-4 errors bits with a high power rate; meaning that this system works well with the lowest BER values.

\section{ACKNOWLEDGMENT}

We would like to express our gratitude for the support of the Islamic University, Imam Ja'afar Alsadiq University, and University of Kufa they provided to us. Their support was the key element for this work to see the light. 


\section{REFERENCES}

[1] M. A. M. Al-Ja'afari, H. M. H. Al-Rikabi, and H. F. M. Fakhruldeen, "Compensation of the Nonlinear Power Amplifier by Using SCPWL Predistorter with Genetic Algorithm in OFDM technique," Journal of Engineering, vol. 24, no. 6, pp. 29-40, 2018, doi: 10.31026/j.eng.2018.06.03.

[2] L. Yang, R. Tian, M. Jia, and F. Li, "A Modified LS Channel Estimation Algorithm for OFDM System in Mountain Wireless Environment," Procedia Engineering, vol. 29, pp. 2732-2736, 2012, doi: 10.1016/j.proeng.2012.01.381.

[3] A. Riadi, M. Boulouird, and M. M. R. Hassani, "Least squares channel estimation of an OFDM massive MIMO system for 5G wireless communications," in International conference on the Sciences of Electronics, Technologies of Information and Telecommunications,vol. 2, pp. 440-450, 2018, doi: 10.1007/978-3-030-21009-0_43.

[4] A. Beydoun and H. Alaeddine, "New fast time synchronization method for MIMO-OFDM systems," in 2018 11th IFIP Wireless and Mobile Networking Conference (WMNC), IEEE, 2018, pp. 1-6, doi: 10.23919/WMNC.2018.8480935.

[5] A. Kumar and M. Magarini, "Symbol error probability analysis of DFrFT-based OFDM systems with CFO and STO in frequency selective Rayleigh fading channels," IEEE Transactions on Vehicular Technology, vol. 68, no. 1, pp. 64-81, 2018, doi: 10.1109/TVT.2018.2881386.

[6] S. Kumar, "Impact of nonlinearities on fiber optic communications," Springer Science \& Business Media, 2011, doi: 10.1007/978-1-4419-8139-4.

[7] R. Tian, Z. Wang, and X. Tan, "A new leakage-based precoding scheme in IoT oriented cognitive MIMO-OFDM systems," IEEE Access, vol. 6, pp. 41023-41033, 2018, doi: 10.1109/ACCESS.2018.2859265.

[8] D. Xu and $\mathrm{H}$. Zhu, "Outage minimized resource allocation for multiuser OFDM systems with SWIPT," IEEE Access, vol. 7, pp. 79714-79725, 2019, doi: 10.1109/ACCESS.2019.2922965.

[9] A. T. Abusabah and H. Arslan, "NOMA for multinumerology OFDM systems," Wireless Communications and Mobile Computing, vol. 2018, 2018, doi: 10.1155/2018/8514314.

[10] J. Hwang, "Simplified Channel Estimation Techniques for OFDM Systems with Realistic Indoor Fading Channels," University of Waterloo, 2009.

[11] M. K. Fadul, D. R. Reising, T. D. Loveless, and A. R. Ofoli, "RF-DNA Fingerprint Classification of OFDM Signals Using a Rayleigh Fading Channel Model," in 2019 IEEE Wireless Communications and Networking Conference (WCNC), IEEE, 2019, pp. 1-7, doi: 10.1109/WCNC.2019.8885421.

[12] K. Dharavathu and M. Anuradha, "Comparison study of linear adaptive equalization filters for image transmission in OFDM systems," International Journal for Research in Applied Science \& Engineering Technology (IJRASET), vol. 6, pp. 758-767, 2018, doi: 10.22214/ijraset.2018.3122.

[13] S. Gauni, C. Manimegalai, K. Kalimuthu, V. Kaushik, and T. R. Rao, "Optical channel analysis of turbo coded MIMO-OFDM system for visible light communication," in Optical And Microwave Technologies: Springer, pp. 161-174, 2018, doi: 10.1007/978-981-10-7293-2_18.

[14] M. Nassiri and G. Baghersalimi, "Comparative performance assessment between FFT-based and FRFT-based MIMO-OFDM systems in underwater acoustic communications," IET Communications, vol. 12, no. 6, pp. 719-726, 2018, doi: 10.1049/iet-com.2017.0776.

[15] A. Munshi and S. Unnikrishnan, "Compressive Sensing in Channel Estimation for SISO and MIMO OFDM Systems," in TENCON 2018-2018 IEEE Region 10 Conference, IEEE, 2018, pp. 0494-0498, doi: 10.1109/TENCON.2018.8650423.

[16] K. Ramadan, M. Dessouky, F. Abd El-Samie, and S. Elagooz, "Virtual quadrature phase shift keying with lowcomplexity equalization for performance enhancement of OFDM systems," AEU-International Journal of Electronics and Communications, vol. 96, pp. 199-206, 2018, doi: 10.1016/j.aeue.2018.08.031.

[17] D. Singh and H. D. Joshi, "Error probability analysis of STBC-OFDM systems with CFO and imperfect CSI over generalized fading channels," AEU-International Journal of Electronics and Communications, vol. 98, pp. 156-163, 2019, doi: 10.1016/j.aeue.2018.10.036.

[18] R. Tang, X. Zhou, and C. Wang, "A Haar wavelet decision feedback channel estimation method in OFDM systems," Applied Sciences, vol. 8, no. 6, p. 877, 2018, doi: 10.3390/app8060877.

[19] J.-J. Van De Beek, O. Edfors, M. Sandell, S. K. Wilson, and P. O. Borjesson, "On channel estimation in OFDM systems," in 1995 IEEE 45th Vehicular Technology Conference. Countdown to the Wireless Twenty-First Century, IEEE, vol. 2, 1995, pp. 815-819, doi: 10.1109/VETEC.1995.504981.

[20] Y. Li, L. J. Cimini, and N. R. Sollenberger, "Robust channel estimation for OFDM systems with rapid dispersive fading channels," IEEE Transactions on communications, vol. 46, no. 7, pp. 902-915, 1998, doi: 10.1109/26.701317.

[21] S. Coleri, M. Ergen, A. Puri, and A. Bahai, "Channel estimation techniques based on pilot arrangement in OFDM systems," IEEE Transactions on broadcasting, vol. 48, no. 3, pp. 223-229, 2002, doi: 10.1109/TBC.2002.804034.

[22] Y. C. Lee, W. C. Jang, U. K. Choe, and G. C. Leem, "The Pilot Alignment Pattern Design in OFDM Systems," arXiv preprint arXiv:1601.07811, 2016.

[23] Mashhadi, Mahdi Boloursaz, and Deniz Gündüz, "Pruning the pilots: Deep learning-based pilot design and channel estimation for MIMO-OFDM systems," IEEE Transactions on Wireless Communications, 2021, doi: 10.1109/TWC.2021.3073309.

[24] Fakhruldeen, Hassan Falah, and Tahreer Safaa Mansour, "Design of Plasmonic NOT Logic Gate Based on Insulator-Metal-Insulator (IMI) waveguides," Advanced Electromagnetics, vol. 9, no. 1, pp. 91-94, 2020, doi: 10.7716/aem.v9i1.1376

[25] Lin, Bin, Bin Lin, Xudong Wang, Weihao Yuan and Nan Wu, "A novel OFDM autoencoder featuring CNN-based channel estimation for internet of vessels," IEEE Internet of Things Journal, vol. 7, no. 8, pp. 7601-7611, 2020, doi: 10.1109/JIOT.2020.2986442. 